\title{
Intelligent aircraft maneuvering decision based on CNN
}

\author{
Bo Li ${ }^{1,2}{ }^{*}$, Shiyang Liang1, Linyu Tian ${ }^{1}$, Daqing Chen ${ }^{3}$ \\ ${ }^{1}$ School of Electronics and Information, Northwestern Polytechnical University, Shanxi Xi'an, China \\ ${ }^{2}$ CETC Key Laboratory of Data Link Technology, Shanxi Xi'an, China \\ ${ }^{3}$ School of Engineering, London South Bank University, London, UK \\ *libo803@nwpu.edu.cn
}

\begin{abstract}
Aiming at the maneuvering decision of aircraft in air combat, an intelligent maneuvering decision model based on convolutional neural network(CNN) is proposed in this paper. Firstly, the situation data, maneuvering decision variables and evaluation indexs are given, and a CNN model that can realize intelligent maneuvering decision is established. Then, according to the evaluation indexes, the structure and parameters of the CNN model are adjusted through the simulation experiments to improve the accuracy and robustness of the maneuvering decision. After that, the validity of the intelligent maneuvering decision model proposed in this paper is verified through comparative experiments that the CNN model can make stable maneuvering decisions with high accuracy. Finally, the flight path in an air combat process is presented.
\end{abstract}

\section{CCS CONCEPTS}

- Computing methodologies $\rightarrow$ Supervised learning by regression - Computing methodologies $\rightarrow$ Neural networks • Applied computing $\rightarrow$ Avionics

\section{KEYWORDS}

Intelligent air combat; maneuvering decision; convolutional neural network; deep learning

\section{Introduction}

Future air war will inevitably develop towards unmanned and autonomous[1]. Autonomous maneuver decision is a critical part to reach a higher level of autonomy and air-combat decision[2,3]. To meet the needs of modern air combat, it is very important to establish a reliable maneuvering decision method, which can make auxiliary decisions to help pilots in manned aircraft and realize autonomous maneuver decision in UAVs. The air combat environment is relative and dynamic, so the decision-making method should be accurate and fast[4].

Many intelligent maneuver decision methods have been formed, such as genetic algorithm[5], expert system method[6] and MCTS Method[7]. Literature[1] realized the maneuvering decision through monte carlo reinforcement learning and showed the flight path and the variation of control variables, which proved the validity of the method. However, this literature only used the position and attitude of both sides to describe the air combat state. The process of air combat is complex, more information should be considered into model such as radar information, missile information.

Deep learning has efficient ability of feature extraction and knowledge expression[8]. In order to realize the intelligent aircraft maneuvering decision, this paper constructs a model using $\mathrm{CNN}$ to fit maneuvering decision variables. The maneuvering decision model takes more comprehensive factors as inputs and directly outputs the change rate of attack angle and the change rate of throttle coefficient, making the decision results more accurate and intuitive.

\section{Convolutional Neural Network}

\subsection{Convolutional Neural Network}

After being trained with known patterns, the Convolutional neural network can learn a lot of mapping relations between input and output without any mathematical expression.

The CNN usually consists of convolutional layers, pooling layers, and fully connected layers $[9,10]$. The essence of convolutional layer is a feature extractor, which can automatically extract the deep information of input data. The pooling layer realizes the sampling processing of the feature map, and keeps useful information while reducing the amount of data. The full connection layer is usually located at the end of the network and can be used for fitting and classification of the features extracted from the previous layers. A common structure of CNN is shown in Figure 1.

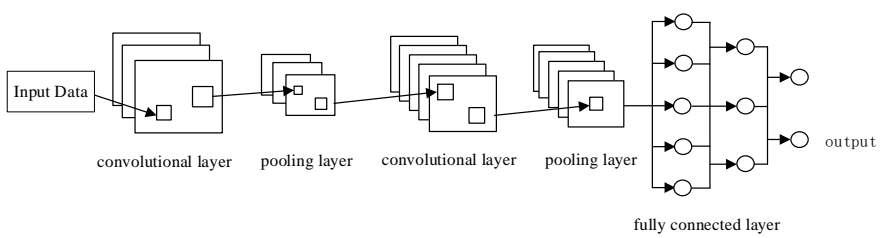

Figure 1: A common structure of CNN

The cores of CNN are the calculation of the convolutional layer and the updating of network parameters. In convolutional layer, local convolution is performed on the feature map of the previous layer by using different convolution kernels. The 
weight of the same convolution kernel is shared, which can reduce the number of parameters. The process of convolution is as follows:

$$
x_{j}^{l}=\sum_{i \in M_{j}} y_{i}^{l-1} \times \omega_{i j}^{l}+b_{j}^{l}
$$

Where $x_{j}^{l}$ is the input of the $j^{\text {th }}$ feature map of the ${ }^{\text {th }}$ layer. $y_{i}^{l-1}$ is the output of the $i^{\text {th }}$ feature map of the $l-1^{\text {th }}$ layer. $\omega_{i j}^{l}$ is the convolution kernel between the $i^{\text {th }}$ feature map of the previous layer and the $j^{\text {th }}$ feature map of the current layer. $b_{j}^{l}$ is the bias

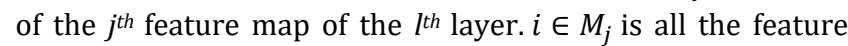
maps of the previous layer that are connected with the $j^{\text {th }}$ feature map of the current layer.

Parameter updating is a process to strengthen the mapping ability of the network. The process includes obtaining the loss function, the back propagation of the error and updating parameters of the network. This paper obtains the loss function according to the follows formula:

$$
E_{d}=\frac{1}{2} \sum_{m=1}^{n}\left(y_{m}-o_{m}\right)^{2}
$$

Where $E_{d}$ represents the loss function. $y_{m}$ represents the output value of output layer, and $o_{m}$ represents truth value.

The back propagation of the error can be realized by calculating the derivative of the convolution kernel and the bias of the loss function for each layer. Finally, the network parameters are updated in some way after calculating the gradient of the network parameters.

\section{Maneuvering decision method based on CNN}

\subsection{Framework of the Maneuvering Decision System}

According to the CNN principle mentioned above, this paper constructs a maneuvering decision model based on CNN and trains the model through the fitting process to the air combat historical dataset. In other words, the nonlinear expression ability of $\mathrm{CNN}$ is utilized to realize the nonlinear mapping from air combat situation to maneuvering decision.

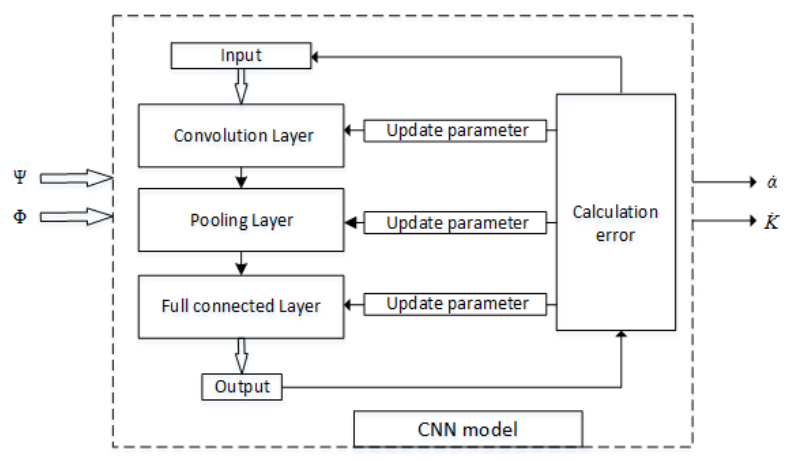

Figure 2: Framework of the Maneuvering Decision System
The Framework of the maneuvering decision system is shown in Figure 2. By inputting our situation data $\Phi$ and enemy's situation data $\Psi$ into the $C N N$ model trained, the maneuvering decision variables including the change rate of attack angle $\dot{\alpha}$ and the change rate of throttle coefficient $\dot{K}$ can be obtained. According to the (3), (4), maneuvering control variables including attack angle $\alpha$, throttle coefficient $K$ can be obtained to realize the maneuvering control of our aircraft.

$$
\begin{aligned}
& \alpha_{t+\Delta t}=\alpha_{t}+\dot{\alpha}_{t+\Delta t} * \Delta t \\
& K_{t+\Delta t}=K_{t}+\dot{K}_{t+\Delta t} * \Delta t
\end{aligned}
$$

Where $t$ represents the current time and $t+\Delta t$ represents the next time.

\subsection{Data Preprocessing}

In the process of air combat, the data of speed, position, acceleration and attitude are generated according to the time sequence. These data describe the state and trend of the aircraft at a certain time and were collected to form the historical dataset.

The size of data collected for different attributes varies greatly, so the dataset must be normalized. In addition, the data sizes at adjacent moments differ slightly. If inputting the training samples in their time order, the gradient may disappear with the back propagation of the error. Therefore, it is necessary to scramble the data.

The formula to normalize the data to the range of $[0,1]$ is as follows:

$$
x_{\text {new }}=\frac{x-\min }{\text { max }-\min }
$$

Where $x$ is the original data. $x_{n e w}$ is the normalized data. $\max$ and $\min$ respectively represent the maximum and minimum of the data.

\subsection{Model Training}

Adam is an adaptive learning rate algorithm[11]. By calculating the first-order moment estimation and second-order moment estimation of each parameter gradient, this algorithm designs an independent adaptive learning rate for each parameter[12]. Compared with other adaptive learning rate algorithms, this algorithm has faster convergence rate, more effective learning and smaller fluctuation of loss function.

Initialize $m_{0}=0, t=0$ and update the parameters $\theta$ according to (6)-(12).

$$
\begin{aligned}
& t=t+1 \\
& g_{t}=\nabla_{\theta} f_{t}\left(\theta_{t-1}\right) \\
& m_{t}=\beta_{1} * m_{t-1}+\left(1-\beta_{1}\right) * g_{t} \\
& v_{t}=\beta_{2} * v_{t-1}+\left(1-\beta_{2}\right) * g_{t}^{2} \\
& \hat{m}_{t}=\frac{m_{t}}{1-\beta_{1}^{t}} \\
& \hat{v}_{t}=\frac{v_{t}}{1-\beta_{2}^{t}}
\end{aligned}
$$




$$
\theta_{t}=\theta_{t-1}-\alpha * \frac{\widehat{m}_{t}}{\left(\sqrt{\widehat{v}_{t}}+\epsilon\right)}
$$

Where $\nabla_{\theta}$ is the gradient of the parameter $\theta . \theta_{t}$ denotes the parameter before the update. $\theta_{t+1}$ denotes the parameter after the update.

\subsection{Evaluation Indexs}

This paper sets mean square error(Mse) and goodness of fitting $\left(R^{2}\right)$ as the evaluation indexs for the validity of model.

\subsubsection{Mean square error}

Mse is the mean squared error between true and predicted values, and it is often used to fully assess the quality of the network. In this paper, it is expressed as:

$$
\begin{aligned}
M s e_{\dot{\alpha}} & =\frac{\sum\left(\dot{\alpha}^{\prime}-\dot{\alpha}\right)^{2}}{n} \\
M s e_{\dot{K}} & =\frac{\sum\left(\dot{K}^{\prime}-\dot{K}\right)^{2}}{n}
\end{aligned}
$$

Where $M s e_{\dot{\alpha}}, M s e_{\dot{K}}$ denote the Mse of the test dataset of the change rate of attack angle and the change rate of throttle coefficient. $\mathrm{n}$ is the group number of test dataset. $\dot{\alpha}, \dot{\alpha}^{\prime}$ are the true value and predicted value of change rate of attack angle. $\dot{K}, \dot{K}^{\prime}$ are the true value and predicted value of change rate of throttle coefficient.

\subsubsection{Goodness of fitting}

Goodness of fitting can be used as the evaluation index of model fitting. The closer it is to 1 , the better the fitting. In this paper, it is specifically expressed as:

$$
\begin{aligned}
& R_{\dot{\alpha}}^{2}=\frac{\sum\left(\dot{\alpha}^{\prime}-\bar{\alpha}\right)^{2}}{\sum(\dot{\alpha}-\dot{\alpha})^{2}} \\
& R_{\dot{K}}^{2}=\frac{\sum\left(\dot{K}^{\prime}-\bar{K}\right)^{2}}{\sum(\dot{K}-\bar{K})^{2}}
\end{aligned}
$$

Where $R_{\dot{\alpha}}{ }^{2}, R_{\dot{K}}{ }^{2}$ denote the goodness of fitting of the test dataset of the change rate of attack angle and the change rate of throttle coefficient. $\overline{\dot{\alpha}}, \overline{\dot{K}}$ represent the mean of the true values of the change rate of attack angle, the change rate of throttle coefficient in the test dataset.

\section{Experiments and Results}

\subsection{Datesets}

In order to train the CNN network effectively, it is necessary to have sufficient data, including training dataset, test dataset.etc. In the one-to-one air combat, an air combat process was set up as a simulation, and the situation information of both sides was recorded in simulation. After several simulations under different initial situations, a total of 16,700 sets of data were collected as the dataset of this paper. Among them, 11700 sets of data were used as the training dataset, and 5000 sets of data were used as the test dataset. The situation information of both sides collected in the air combat are as follows.

\subsubsection{The situation information of our side}

The situation information of our side includes simulation information, survival information, position information, speed information, acceleration information, attitude information, control information, target information, sensor information, radar information and missile information.

\subsubsection{The situation information of the other side}

It is difficult to obtain all the situation information of the enemy in the actual air combat. This paper selectes the position information and speed information of aircraft that can be obtained as situation information of the other side.

\subsection{Optimization Experiments}

\subsubsection{Training and Testing}

The optimization experiments included two sets of experiments. CNN models in the table 2 were used to fit the change rate of attack angle and the change rate of throttle coefficient respectively in the two sets of experiments. After training, the test dataset was input into the model to obtain the predicted value. Mse and $R^{2}$ were calculated by comparing the predicted value with the true value. After that, the best model was selected.

In this experiment, the training was carried out for a total of 5,000 rounds. The batchsize was 512 . The activation function was tanh function. The optimized method was Adam, and the parameters in this method were set as $\alpha=0.001, \beta_{1}=0.9$, $\beta_{2}=0.999, \in=10 e-8$.

Table 2: CNN models

\begin{tabular}{lll}
\hline CNN Models & Convolution layers & Hidden layers \\
\hline M1 & 2 & 2 \\
\hline M2 & 3 & 2 \\
\hline M3 & 3 & 4 \\
\hline M4 & 4 & 3 \\
\hline
\end{tabular}

\subsubsection{Results and Analysis}

Table 3 shows the evaluation indexs for different CNN models.

Table 3: The evaluation indexs for different CNN models.

\begin{tabular}{lrrrr}
\hline CNN Models & $M s e_{\dot{\alpha}}$ & $R_{\dot{\alpha}}{ }^{2}$ & $M s e_{\dot{K}}$ & $R_{\dot{K}}{ }^{2}$ \\
\hline M1 & $0.99 \%$ & $88.9 \%$ & $2.5 \%$ & $70.2 \%$ \\
\hline M2 & $0.95 \%$ & $87.9 \%$ & $2.4 \%$ & $80.1 \%$ \\
\hline M3 & $0.91 \%$ & $89.7 \%$ & $1.96 \%$ & $82.5 \%$ \\
\hline M4 & $1.06 \%$ & $87.9 \%$ & $2.50 \%$ & $69.7 \%$ \\
\hline
\end{tabular}

By analyzing $M s e$ and $R^{2}$, it can be concluded that M3 reached the optimum when fitting the change rate of attack angle and the change rate of throttle coefficient. In this paper, BP neural 
network and CNN(M3) are compared in comparative experiments. The structure of M3 is shown in table 4.

Table 4: The structure of M3

\begin{tabular}{lllll}
\hline \hline Layer & Type & Kernel size & Filters & Output Size \\
\hline \hline I & Input & & & $7 \times 7 \times 1$ \\
\hline C1 & Convolution & $2 \times 2$ & 10 & $6 \times 6 \times 10$ \\
\hline C2 & Convolution & $2 \times 2$ & 10 & $5 \times 5 \times 10$ \\
\hline C3 & Convolution & $3 \times 3$ & 10 & $3 \times 3 \times 10$ \\
\hline F1 & Fully connected & & 50 \\
\hline F2 & Fully connected & & 30 \\
\hline F3 & Fully connected & & 10 \\
\hline F4 & Fully connected & & 1 \\
\hline O & Output & & 1 \\
\hline
\end{tabular}

\subsection{Comparative Experiments}

\subsubsection{Training and Testing}

The comparative experiments included four groups of experiments. The first group of experiments used BP neural network to fit the change rate of attack angle. The second group used the optimal CNN model in optimization experiments to fit the change rate of attack angle. The third group of experiments used BP neural network to fit the change rate of the throttle coefficient. The fourth group of experiments used the optimal CNN model in optimization experiments to fit the change rate of the throttle coefficient. The structure of the BP neural network and CNN used in comparative experiments is shown in Table 5.

The losses in the process of model training were recorded and used as the evaluation basis for the convergence rate of the model. The evaluation indexs of comparative experiments were the convergence rate of training, Mse and $R^{2}$ of the test dataset.

In this experiment, the training was carried out for a total of 5,000 rounds. The batchsize was 512 . The activation function was tanh function. The optimized method was Adam, and the parameters in this method were set as $\alpha=0.001, \beta_{1}=0.9$, $\beta_{2}=0.999, \epsilon=10 e-8$.

Table 5: Comparison of the structure of BP neural network and CNN

\begin{tabular}{lll}
\hline \hline & Convolution layers & Hidden layers \\
\hline \hline BP neural network & & 7 \\
\hline CNN & 3 & 4 \\
\hline
\end{tabular}

4.3.2 Comparison and Analysis of Convergence Speed Between BP Neural Network and CNN

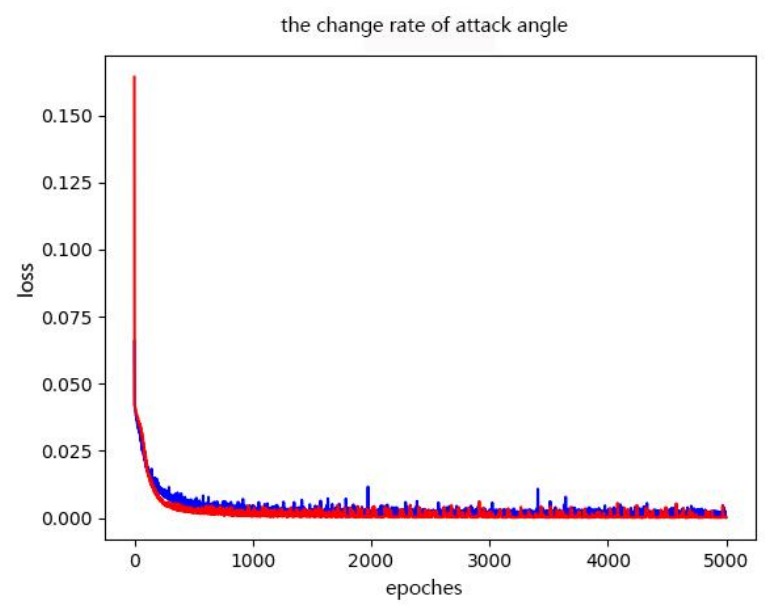

Figure 3: Training losses of BP neural network and CNN in the 5000 epochs of training are shown. The red represents the $\mathrm{CNN}$. The Blue represents the $\mathrm{BP}$ neural network.

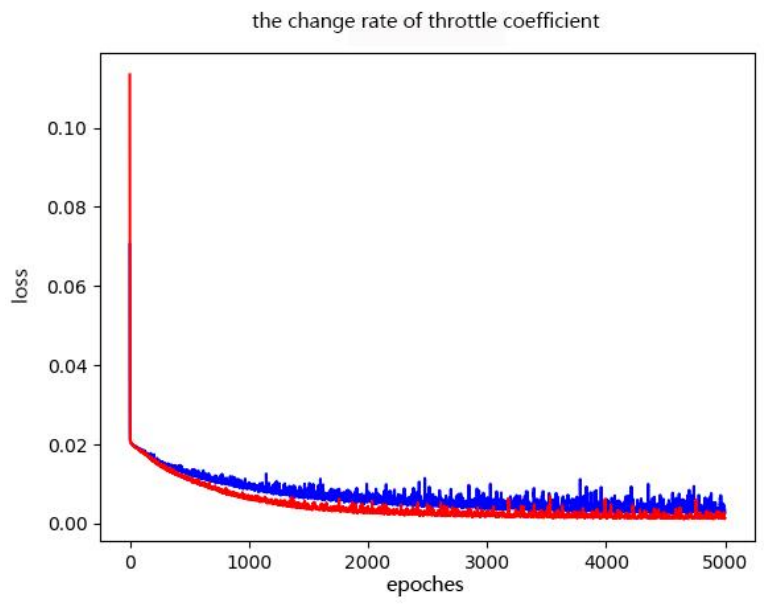

Figure 4: Training losses of BP neural network and CNN in the 5000 epochs of training are shown. The red represents the $\mathrm{CNN}$. The Blue represents the $\mathrm{BP}$ neural network.

Figure 3 shows the comparison of convergence speed between BP neural network and CNN when fitting the change rate of attack angle. Figure 4 shows the comparison of convergence speed between BP neural network and CNN when fitting the change rate of throttle coefficient. From the experimental results, it can be seen that the $\mathrm{CNN}$ achieved small errors in the process of fitting. Meanwhile, compared with BP neural network, CNN had faster convergence speed and smaller and more stable losses in training.

4.3.3 Comparison and Analysis of Evaluation Indexes Between BP Neural Network and CNN 
Table 6: The evaluation indexes when fitting the change rate of attack angle

\begin{tabular}{l|l|c}
\hline & $M s e_{\dot{\alpha}}$ & $R_{\dot{\alpha}}{ }^{2}$ \\
\hline BP neural network & $1.15 \%$ & $86.81 \%$ \\
\hline CNN & $0.73 \%$ & $90.87 \%$ \\
\hline
\end{tabular}

Table 7: The evaluation indexes when fitting the change rate of throttle coefficient

\begin{tabular}{l|c|c}
\hline & $M s e_{\dot{K}}$ & $R_{\dot{K}}{ }^{2}$ \\
\hline BP neural network & 0.024095 & $64.23 \%$ \\
\hline CNN & 0.019584 & $82.53 \%$ \\
\hline
\end{tabular}

For the two kinds of maneuvering decision variables, the Mse and $R^{2}$ in tables indicate that the maneuvering decision model constructed by CNN achieved high fitting accuracy. Meanwhile, compared with BP neural network, the maneuvering decision models constructed by CNN had higher fitting accuracy and higher validity.

\subsection{The Flight Path of an Air Combat}

After models of maneuvering decision were established, they were applied to the simulation of air combat. In the simulation, our side used the CNN model to make maneuvering decisions, while the other side used the original method. A result of air combat is shown as figure 5 .

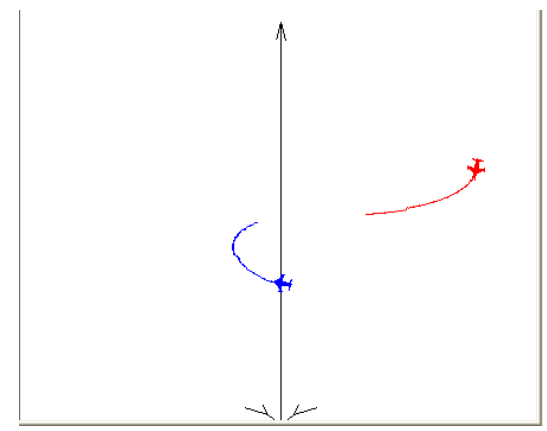

Figure 5: Flight path of two sides in 3D.

In figure 5, the red fighter represents our side and the blue fighter represents the other side. In the initial state, the red fighter and the blue fighter have the same weapons and aircraft type. Figure 5 shows that the red fighter made a dive to approach and attack the blue fighter.

\section{Conclusion}

According to the maneuvering decision problem of aircraft in air combat, this paper constructs a maneuvering decision model by using the powerful feature extraction ability and reducing the over-fitting ability of the CNN. By training a large amount of air combat data, the model can directly extract the internal relationship between combat situation data and maneuvering decision variables. The experimental results in this paper show that the model is accurate in maneuvering decision. Meanwhile, the CNN has better performance, stronger generalization ability and higher fitting accuracy in maneuvering decision compared with BP neural network.

In this paper, the maneuvering decision model outputs the maneuvering decision variables directly in the decisionmaking process, and then the maneuvering control can be realized. This makes the decision-making results more intuitive and effective, and also provides ideas for further research.

\section{ACKNOWLEDGMENTS}

This research was supported by The Aeronautical Science Foundation of China (Grant No.2017ZC53021) and The Open Project Fund of CETC Key Laboratory of Data Link Technology (Grant No. CLDL-20182101).

\section{REFERENCES}

[1] Minglang Cui, Haiwen Du, Zhenglei Wei and Qingpeng Sun(2018). Maneuvering decision in short range air combat for unmanned combat aerial vehicles. Chinese Control And Decision Conference (CCDC), Shenyang, 1783-1788.

[2] Changqiang Huang, Kangsheng Dong, Hanqiao Huang, Shangqin Tang and Zhuoran Zhang(2018). Autonomous air combat maneuver decision using Bayesian inference and moving horizon optimization. Journal of Systems Engineering and Electronics, 29(01):86-97.

[3] Li Fu, Fuhuai Xie, Dongzheng Wang and Guanglei Meng (2014). The overview for UAV Air-Combat Decision method. The 26th Chinese Control and Decision Conference (2014 CCDC), Changsha, 3380-3384.

[4] Changqiang Huang, Kexin Zhao, Bangjie Han and Zhenglei Wei(2018). Maneuvering Decision-making Method for UAV Based on Approximate Dynamic Programming. Journal of Electronics \& Information Technology, 40(10):2447-2452.

[5] Shuo Ku, Dali Ding, Changqiang Huang, Jie Wang and Cong Li(2018) Optimal tactical maneuver control model based on improved genetic algorithm. Fire Control \& Command Control, 43(12):21-26.

[6] Li Fu, Fuhuai Xie, Guanglei Meng and Dongzheng Wang(2015). An UAV air combat decision expert system based on receding horizon control Journal of Beijing University of Aeronautics and Astronautics, 41(11):1994-1999.

[7] $\mathrm{Xu} H \mathrm{He}$, Xiaoning Jing and Chao Feng(2018). Air Combat Maneuver Decision Based on MCTS Method. Fire Control \& Command Control, 43(3):34-39.

[8] Xiaohai Zhang, Xinwen Cao, Songtao Geng and Yanli Zhang(2018) Research on intelligent decision making of military assistance based on deep learning. Journal of weapon equipment engineering, 39(10):162167.

[9] Shengxiang Tao and Lei $\mathrm{Ai}(2008)$. Application and realization of convolutional neural network in digital gun pointing system. Journal of weapon equipment engineering, 39(07):145-150.

[10] Saad Albawi, Tareq Abed Mohammed and Saad Al-Zawi(2017) Understanding of a convolutional neural network. International Conference on Engineering and Technology (ICET), Antalya, 1-6.

[11] Wangwang Zhou, Peiyang Yao, Jieyong Zhang, Xun Wang and Shuai Wei (2018). Combat Intention recognition for aerial targets based on deep neural network. Acta Aeronautica et Astronautica Sinica, 39(11):32468.

[12] Diederik P.Kingma and Jimmy Lei Ba(2015). Adam:A Method for Stochastic Optimization. arXiv:1412.6980v9. Retrieved from https://arxiv.org/abs/1412.6980v9 